\title{
Does undergraduate teaching of infection prevention and control adequately equip graduates for medical practice?
}

\author{
${ }^{1}$ Department of Paediatrics and Child Health, Stellenbosch University, Cape Town, South Africa \\ ${ }^{2}$ Division of Community Health, Stellenbosch University, and Western Cape Government Health, Cape Town, South Africa \\ ${ }^{3}$ Division of Community Health, Stellenbosch University, Cape Town, South Africa \\ ${ }^{4}$ Unit for Infection Prevention and Control, Division of Community Health, Stellenbosch University, Cape Town, South Africa \\ * Sade Adeniyi, Neil Cameron, Lilian Dudley, Charlyn Goliath, Fidele Mukinda, Anke Rohwer, Taryn Young
}

A Dramowski, ${ }^{1}$ MMed Paed; F Marais, ${ }^{2} \mathrm{PhD}$; B Willems, ${ }^{3} \mathrm{MB}$ ChB; S Mehtar, ${ }^{4}$ FCPath (SA) and the SURMEPI curriculum review working group ${ }^{*}$

Corresponding author: A Dramowski (dramowski@sun.ac.za)

Background. Knowledge, skills and desirable clinical practices in infection prevention and control (IPC) should be acquired during undergraduate medical training. Although knowledge and skills are learnt in the formal curriculum, attitudes and practices are assimilated by observation and modelling. We investigated whether undergraduate teaching and learning of IPC at Stellenbosch University adequately prepared graduates for medical practice. Methods. A situational analysis of IPC teaching was conducted, including development of IPC competencies, a curriculum review, an email survey of MB,ChB graduates and semistructured focus group or personal interviews with teaching faculty. Qualitative data were assessed using a framework analysis approach. Results. All graduate survey respondents who completed the IPC-related questions $(n=180)$ agreed that teaching of IPC was important and most (156; 87.8\%) felt that IPC teaching had adequately prepared them for practice. Despite this perception, graduates encountered difficulty implementing IPC best practice owing to lack of management support for IPC and resource constraints. Faculty members disagreed regarding the adequacy of IPC teaching and some were concerned that the curriculum failed to prepare graduates for medical practice. Graduates and faculty felt strongly that undergraduate IPC teaching and learning could be improved by addressing suboptimal IPC practices and lack of clinician role models for IPC at training institutions. Conclusion. IPC knowledge transfer appears adequate in most competency areas. However graduates struggled to implement IPC best practice in the clinical field. Undergraduate IPC teaching and learning could be enhanced by development of clinician role models for IPC and strengthened IPC practices in training institutions.

Afr J Health Professions Educ 2015;7(1 Suppl 1):105-110. DOI 10.7196/AJHPE.500

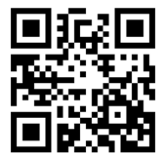

Infection prevention and control (IPC) is a discipline that aims to prevent or control infection transmission in healthcare facilities and the community. ${ }^{[1]}$ In South African (SA) healthcare facilities IPC is gaining increasing importance, forming one of six key priorities in the National Core Standards for Health Establishments. ${ }^{[2]}$ Medical graduates' IPC knowledge base, attitudes and practices are established at undergraduate level..$^{[3]}$ Although knowledge and skills are acquired through the taught curriculum, attitudes and practices are more often modelled on those of medical faculty and senior colleagues. ${ }^{[4]}$ In many medical curricula, IPC is not taught as a stand-alone subject but rather as a 'golden thread' throughout undergraduate training. At Stellenbosch University, IPC-related content is included within modules including microbiology, infectious diseases, clinical disciplines and clinical skills laboratory training. A situational analysis was conducted to assess the content, coverage and opportunities for enhancement of IPC teaching within the Stellenbosch University's $\mathrm{MB}, \mathrm{ChB}$ curriculum. The analysis included: development of IPC competencies for $\mathrm{MB}, \mathrm{ChB}$ students; a curriculum module review; a survey of recent $\mathrm{MB}, \mathrm{ChB}$ graduates and interviews with teaching faculty. The curriculum research was an activity of the Stellenbosch University Rural Medical Education Partnership Initiative (SURMEPI) and included four undergraduate knowledge areas: IPC, evidence-based health, Public Health and Health Systems and Services Research. This paper reports on medical graduates' post-qualification experiences of IPC, as well as graduates' and faculty perceptions of teaching and learning in IPC.

\section{Methods \\ IPC competencies}

Key and enabling IPC competencies for Stellenbosch University MB,ChB students (Table 1) were developed and refined in consultation with faculty members and IPC expert practitioners, drawing on the CanMEDS framework $^{[5]}$ and international literature. This Canadian-developed framework is widely used to define required competencies for medical doctors in each of seven 'roles' including: medical expert, communicator, collaborator, manager, health advocate, scholar, and professional.

\section{Graduate survey}

An electronic questionnaire (distributed between January and June 2012) surveyed recent Stellenbosch University medical graduates (2004-2010). Likert scales and open-ended questions explored graduates' opinions of the appropriateness, acceptability and relevance of undergraduate IPC teaching and learning, and their experiences of IPC practice post graduation. Data were analysed using SPSS statistical software version 20 (quantitative data) and framework analysis aided by ATLAS.ti version 7.0 (qualitative data). One author coded all transcripts, while a second author coded $25 \%$ of the same data independently. There was more than $80 \%$ agreement between authors and we thus relied on the coding of the first author. We grouped codes into emerging themes for each question. Comments from survey respondents who answered in Afrikaans were translated into English. Ethical approval was obtained from the Stellenbosch University Health Research Ethics Committee (S11/10/004). 
Table 1. Key competencies in infection prevention and control (IPC) for Stellenbosch University medical graduates

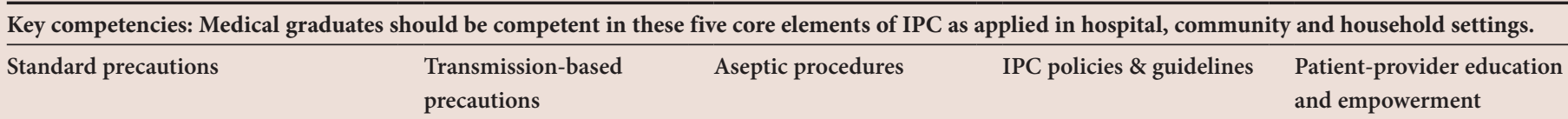

Must be able to apply and adhere to the correct procedure-based standard precautions in all clinical practices

\section{Hand hygiene}

Personal protective equipment use

Safe disposal of sharps

Injection safety

Decontamination of equipment

Clinical waste management
Must be able to implement and adhere to the appropriate transmissionbased precautions for the prevention of infection
Must apply the correct aseptic procedure when using or inserting medical devices to prevent infection
Must understand the importance and implications of local IPCrelated legislation and requirements
Must understand the importance and benefits of IPC knowledge transfer between patients and healthcare staff, and possess the necessary skills in education and communication

General IPC measures Prevention of diarrhoeal and respiratory disease TB infection control Advocacy
Central venous lines

Endotracheal intubation

Suturing of minor wounds Delivery of babies

Assisting with surgical operations

\section{Notification of}

communicable disease EPI surveillance system Reporting of occupational risks/exposures Exposure to blood and body fluids (sharps and splashes)

Enabling competencies: Medical graduates should have working knowledge of infectious disease pathogenesis, basic epidemiology, laboratory investigations, antimicrobial chemotherapy, prophylaxis and vaccination

EPI = expanded programme on immunisation, with surveillance including acute flaccid paralysis, neonatal tetanus, measles and adverse events following immunisation.

\section{Faculty interviews}

Stellenbosch University faculty were involved in $\mathrm{MB}, \mathrm{ChB}$ teaching were identified from a list of undergraduate module (subject) convenors. Nine proposed focus groups were created, according to content areas covered and/or module placement in the curriculum. Each participant (module convenor) was informed of the study purpose, provided written consent and received documents in advance: the IPC key competencies for undergraduates (Fig.1); the IPC curriculum module review findings; and the graduate survey report. Trained interviewers captured all interview sessions on a digital voice recorder, with additional field notes. Qualitative data were transcribed (with participants de-identified) followed by framework analysis, ${ }^{[6]}$ aided by ATLAS. ti version 7.0 software. A master code list (Appendix 1) was developed through discussion and consensus by the larger SURMEPI curriculum review working group. After all transcripts were reviewed, emerging themes, sub-themes and cross-cutting themes were identified. The coding process was conducted as described above. Ethical approval was obtained from the Stellenbosch University Health Research Ethics Committee (N12/11/081).

\section{Results}

\section{Graduate survey}

A total of 375 of 980 recent medical graduates (38\%) responded to the survey invitation,

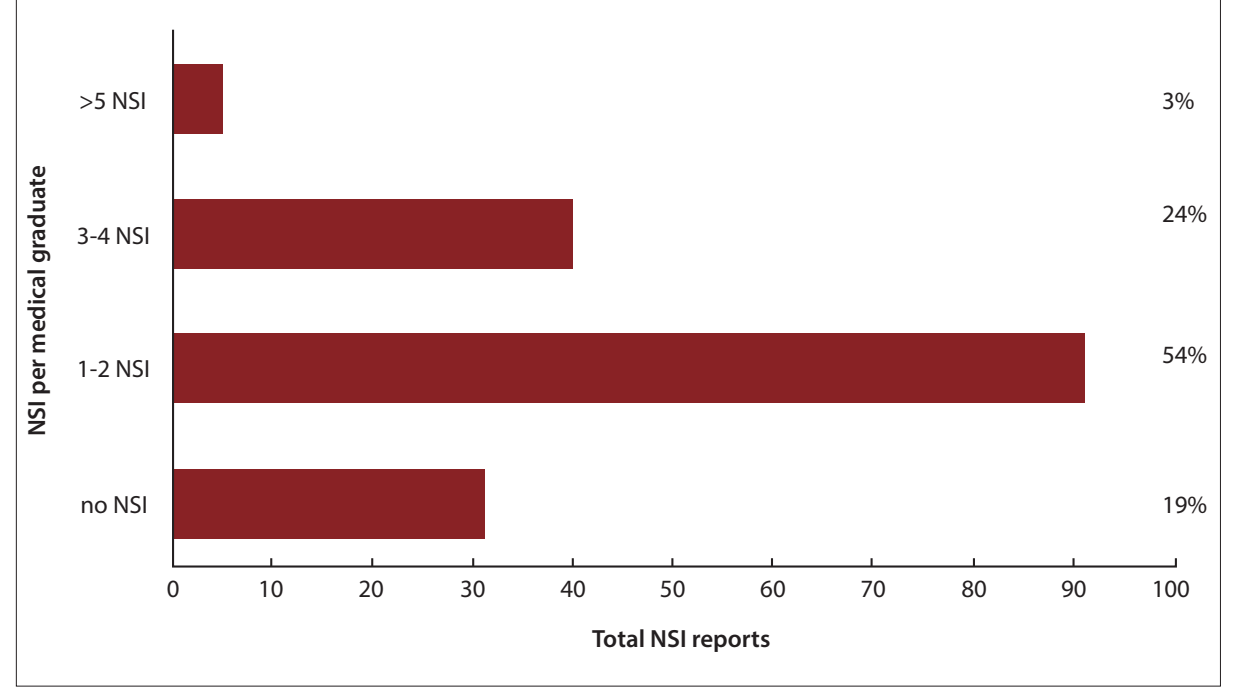

Fig. 1. Needle-stick injuries (NSI) among recent medical graduates ( $n=167)$. Not all survey respondents completed all sections of the survey and therefore participant numbers vary by question.

although only $180(18.4 \%$ of the target population) completed all IPC-related questions (Table 2). Partially completed surveys were included in the analysis. All IPC section respondents $(n=180)$ agreed it was important to learn IPC at undergraduate level. Most (156; 87.8\%) felt IPC teaching had adequately prepared them for medical practice. From a list of ten IPC competencies, most respondents $(103 / 180 ; 57.2 \%)$ felt the topics had received adequate or comprehensive coverage in the curriculum (Table 3). Topics reported as inadequately covered were: occupational health; assessment of IPC policies/guidelines; and leadership/management in IPC. Despite reports that training on the topic of sharps management was adequate, needle-stick injuries were common (Fig. 1). Graduates' qualitative responses are included with the faculty interviews below. 


\section{Faculty interviews}

A total of 15 interviews were conducted: 20 participants completed 5 focus group interviews (2 - 7 participants each) and 10 completed individual interviews (as they were unable to attend their focus group date.)

\section{Qualitative responses from the graduate survey and faculty interviews}

The themes emerging from the graduate survey and faculty interviews were remarkably similar. Pertinent verbatim quotations appear below grouped in emerging themes and coded with unique participant numbers for graduates (G) and faculty (F).

All graduates and many faculty members reported that IPC was an essential and relevant subject to their discipline, as well as the greater SA healthcare context:

- F14 'Infection prevention must be part of what we do every day. It is absolutely essential especially in South Africa ...'

- F15 '[IPC is]...probably one of the cornerstones particularly in our country ... so we're very cognisant of infection control ... I think that would be very relevant to our students.'

Although many faculty members agreed that the subject of IPC was relevant, there was disagreement as to how adequately it was addressed in the curriculum:

- F05 'At undergraduate level we don't really provide the basic principles of infection prevention and control. We do provide some specific principles as it applies to our discipline.'

- F11 'Well in no part of my teaching do I do infection control at all and .... we probably should?

- F08 'Infection control $\ldots$ that definitely is addressed very well ... previously it wasn't like that'.

Despite reporting that IPC was generally well taught, many graduates and faculty pointed out that IPC-related knowledge was seldom implemented in daily practice. Graduates and faculty reported that this 'know-do' gap was perpetuated by a failure of clinicians in training institutions to model good IPC practice:

- F04 'It is almost like we see it merely as a principle and that is where it stays... [IPC is] taught as principles but there is very little translation of these principles when they start in their clinical rotations...'
- G18 'Do as I say, not as I do. We had quite a few theoretically sound lectures, but none of those measures were applied consistently in practice - particularly relating to TB infection control, the examples set were disastrously inadequate and even misleading.

- F09 'Then they get this hidden curriculum ... where we teach things in the lecture halls and then in the hospital they get told N95 masks are for sissies. You don't need a mask, just get out, just do the work... they see seniors not wearing gloves when they do invasive procedures, not washing their hands and so what we teach and what they see are not the same.'

- F03 'We can teach them whatever we want. I think many students follow what is modelled?

Table 2. Graduate survey participant demographics $(N=287)^{*}$

\begin{tabular}{ll}
\hline Year MB,ChB degree obtained & $\boldsymbol{n}$ (\%) \\
\hline 2004 & $31(10.8)$ \\
2005 & $53(18.5)$ \\
2006 & $50(17.4)$ \\
2007 & $31(10.8)$ \\
2008 & $39(13.6)$ \\
2009 & $40(13.9)$ \\
2010 & $43(15.0)$ \\
Current employment position & $\boldsymbol{n}(\%)$ \\
Internship & $41(14.2)$ \\
Community service & $39(13.5)$ \\
Medical officer & $82(28.5)$ \\
General practitioner & $38(13.2)$ \\
Registrar & $70(24.3)$ \\
Specialist & $3(1.0)$ \\
Other & * Not all survey respondents completed all sections of the survey and therefore participant numbers \\
for each question vary. \\
Oother $=$ other medical-related post, other non-medical profession, unemployed.
\end{tabular}

Table 3. Graduates' opinion of IPC competency area coverage in the undergraduate MB,ChB curriculum

\begin{tabular}{llll}
\hline Competency area $^{*}$ & $\begin{array}{l}\text { Not at all/ inadequate } \\
\boldsymbol{n}(\%)\end{array}$ & $\begin{array}{l}\text { Basic } \\
\boldsymbol{n}(\%)\end{array}$ & $\begin{array}{l}\text { Adequate/ comprehensive } \\
\boldsymbol{n}(\%)\end{array}$ \\
\hline Hand hygiene & $9(5)$ & $23(12.7)$ & $149(82.3)$ \\
Use of personal protective equipment & $14(7.8)$ & $32(17.9)$ & $133(74.3)$ \\
Sharps management/needle-stick injury & $8(4.5)$ & $14(7.9)$ & $156(87.6)$ \\
Decontamination of equipment between patient use & $20(11.1)$ & $41(22.9)$ & $118(66.6)$ \\
Clinical waste management & $35(19.4)$ & $52(29)$ & $93(51.6)$ \\
Transmission-based precautions & $19(10.8)$ & $40(22.6)$ & $118(66.6)$ \\
Prevention of infection transmission during procedures & $14(7.9)$ & $27(15.3)$ & $136(76.8)$ \\
Assessment of IPC policy/guidelines for healthcare facilities & $51(28.5)$ & $71(39.7)$ & $57(31.8)$ \\
Motivation of HCW to adhere to IPC policies/guidelines & $50(28.2)$ & $67(37.9)$ & $60(33.9)$ \\
Occupational health and HCW rights to a safe work environment & $62(34.4)$ & $71(39.4)$ & $47(26.2)$ \\
* Not all survey respondents completed all sections of the survey and therefore participant numbers for each question vary. & &
\end{tabular}


- G82 'The training in IPC is good, but healthcare workers are not afraid enough of contracting TB, so they don't implement precautions... our senior consultants and registrars don't set a good example'.

Many faculty members felt that the curriculum did not adequately prepare graduates for practice in the SA context, and in particular that the training provided failed to equip graduates with skills to implement health system change.

- F09 'They know all the fancy stuff, but the simple things that they need to do in practice they haven't been taught.'

- F06 'They need to be prepared for a severely resource-constrained area ... they need to have an attitude of how to adapt and to compromise, which maybe we are not preparing them for ...'

- F01 'We teach students how the health system works, but okay, so what? The question is how do I improve the health system, how do I engage, how do I really make a change? ... they don't see that ... in practice.'

- F09 'I think we need to focus more on making the students aware that when they go out with that degree that they should become the so-called change agents. They don't see themselves as being able to make a difference in public health sector in that way.

This perception was confirmed by graduates' reports of challenges in implementing IPC best practice in SA healthcare facilities. Graduates singled out facility managers for not providing adequate support for IPC, particularly lack of provisions and failure to implement IPC policies and best practice. Resource constraints, including poor planning of facilities (especially isolation areas and ventilation) were commonly cited issues.

- G45 'Weak management with the attitude that "you must just make do with what is available" - even if it is not the right way to practise.'

- G66 'Managers have limited insight into IPC matters, and are often ineffective and underqualified.

- G18 'We have no recourse to improve matters or at the very least protect ourselves. Administrators and peers are guilty of gross neglect of self and colleagues.

- G54 'A breakdown in communication between managers and clinicians prevents IPC problems being addressed.

- G63 'There are no isolation rooms available ...'

- G30 'The most basic IPC provisions and protective equipment are not available ... either due to budget constraints or mismanagement in the ordering of stock.

- G66 '[There is] extremely poor planning of ventilation in healthcare facilities with a high TB burden.

Graduates were particularly concerned regarding the attitude of senior colleagues to occupational health and patient safety. They reported a general disregard of safety in the healthcare environment and a lack of accountability for deviation from IPC policies.

- G178 'Work-related injury is something most doctors encounter at least once in their careers, whether it's HIV, hepatitis exposure or TB; I was amazed how much emotional stress these injuries create and worried by how little support there is for affected doctors.'

- G18 'Doctors are forced to work in dangerous situations with unsafe sharps containers, lack of gloves, absence of N95 respirators, isolation facilities or adequate ventilation.

- G05 'Fellow staff and management disregard the importance of IPC; there is just an attitude of: no one cares and it's nobody's responsibility'.
- G120 'There are no consequences for ignoring IPC policies'.

Given these realities of healthcare practice, how should the curriculum be adapted to better prepare medical graduates? Faculty identified multiple barriers to inclusion of more IPC-related content in the MB, ChB curriculum (grouped thematically into curriculum-related and practice-environmentrelated challenges).

\section{Curriculum challenges}

- F15 'There is so little time so you are trying to do the best in that little time and impart as much of your discipline as you can. So you sort of prioritise on the important ... and hope that somebody else will catch up somewhere along the line.'

- F01 'You never talk about infection [IPC], you don't demonstrate it ... it is not our responsibility. I just look after my small piece, you understand. So there is a big motivation in change management necessary for ... academics within our faculty, to become role models themselves, to integrate these things themselves.'

- F01 'The challenge for the curriculum which contains these four components [IPC], is not just to integrate it into the curriculum because in a sense it is very, very difficult. It's how to integrate it within the clinical care of a patient.

\section{Practice environment challenges}

- F06 ... challenging things like infection prevention ... we know what is the right thing to teach the student ... but they will come and tell you tomorrow, when I get to the hospital this is not there. What must I do? Then you teach them the next best thing which you know is not the right thing, and then the irony is, even when the best thing is there, they do the thing that's wrong.'

- F04 '... groups of the students more and more mention of the lack of infection control in the hospitals or the disregard to [of] established protocols.'

- F09 'We must train more medical students, wards are crowded, patients are overexamined, not enough staff to properly teach.'

Despite these many challenges, faculty and graduates identified several opportunities for improvement of the IPC curriculum, and were generally supportive of integrating IPC teaching within other disciplines.

- F06 'There are a lot of opportunities, we don't necessarily use all of them but we try ... if you teach a skill, obviously watch the infection prevention control related to that ...'

- F14 ' ... formal teaching is not good enough, we need to demonstrate, we need to be actively involved to be role models for students to actually see what we do in clinical practice.'

- G122 'Empower students to address IPC issues - most training takes place in the clinical rotations and through the example set by senior staff. IPC guidelines must be effectively applied in the training institutions where students will learn either good or bad practices.'

- G50 'Incorporate IPC into every practical exam. If you do not wash your hands or take the correct precautions - then you fail or get marked down.'

- G05 'Improve the training environment - the actual hospitals and clinics. Teach students to address IPC shortcuts in facilities assertively... if a student learns good IPC during clinical rotations lectures might even be unnecessary!? 


\section{Discussion}

Graduates' universal agreement on the importance of undergraduate IPC teaching is unsurprising given SA's high burden of communicable disease $^{[7]}$ and daily exposure of young doctors to infectious diseases. Many respondents argued that IPC should receive greater emphasis in the curriculum, possibly indicating that at student level, the relevance of IPC to one's future practice is underestimated.

It is possible that recall bias may be present among graduates asked to comment on their experience of IPC teaching several years ago. Notwithstanding this limitation, graduates were largely in agreement on areas of deficiency in the IPC curriculum. The 'inadequately covered' IPC topics (occupational health, IPC policies/guidelines, leadership/management in IPC) are all core competencies needed for medical practice. Graduates reported extreme dissatisfaction with healthcare management and the lack of resources available for IPC. This perception is not unfounded as a recent baseline audit measured IPC compliance in South African healthcare facilities at only $50 \% .{ }^{[8]}$

Although the vast majority of graduates agreed sharps management was well covered in the IPC curriculum, needle-stick injuries were common (with $81.4 \%$ of respondents reporting one or more injury since graduation). In a similar cohort of young doctors in the USA, early career sharps exposures were reported from 103 of 455 healthcare workers $(22.6 \%){ }^{[9]}$ It is well-demonstrated that knowledge alone will not necessarily alter practice. However, additional data are needed to explore potential contributing factors to the high sharps injury rate, e.g. fatigue, patient load, lack of sharps bins, unavailability of safety-engineered devices and recapping of needles.

Although over $80 \%$ of graduates reported that the IPC curriculum prepared them adequately for medical practice, the qualitative data were contradictory. Graduates appeared ill-equipped to deal with challenges in SA healthcare facilities including ineffective management, resistance to change and lack of resources/basic provisions for IPC. Given the nature of these challenges, curriculum enhancement alone is unlikely to improve graduates' ability to address IPC practice issues. $\mathrm{MB}, \mathrm{ChB}$ graduates require generic skills to plan, implement, manage and measure health-related interventions. These so-called 'soft skills' of advocacy, healthcare management and communication (all prioritised in the CanMEDS model) would empower graduates to address IPC (and other quality improvement) issues. It is clear that graduates have the relevant knowledge to identify problems, but lack the skills and attitude to effect change. Enhanced skills in advocacy, monitoring and evaluation and healthcare management (although not uniquely applicable to IPC) would better equip graduates to promote systems, attitudinal and behaviour change at their facilities. Teaching of this skill set could be integrated in the clinical disciplines and emphasised within a new module called 'Doctor as Change Agent' in the MB,ChB V curriculum.

Role-modelling, the process in which 'faculty members demonstrate clinical skills and manifest professional characteristics ${ }^{\text {[10] }}$ has a broad influence on undergraduate learning, including students' attitudes and behaviours (both positive and negative). ${ }^{[11,12]}$ Although faculty members concurred that IPC was highly relevant, in almost all instances they did not incorporate IPC principles in their teaching. Graduates noted that although faculty taught them the correct IPC principles, these same 'clinician role models' demonstrated contradictory attitudes and behaviours in daily practice. A negative institutional climate with widespread examples of undesirable IPC practice at their training facilities influenced students' perceptions of IPC. The curriculum redevelopment process should acknowledge and address shortcomings in IPC practice at universityaffiliated training facilities as a priority.

Graduates and faculty supported the idea of integrating IPC within other disciplines. Given the shortage of dedicated faculty to teach IPC and the 'crowded' curriculum, incorporation of IPC principles relevant to each clinical discipline would seem a practical solution. This would require recruitment of 'IPC champions' to lead the process of integration at departmental level. Acknowledgement of IPC as an essential competency in all clinical disciplines should encourage faculty to become better role models and advocates for improved IPC practice in training institutions. This approach could (without the need for further didactic IPC teaching) produce young doctors who will perpetuate the IPC best practices they observed during undergraduate training.

\section{Conclusion}

Medical graduates and faculty agree on the relevance and importance of IPC teaching in the MB,ChB curriculum. Graduates feel that most IPC topics are adequately taught, preparing them well for medical practice. However, graduates and faculty report challenges in IPC best practice implementation including lack of leadership support and resource constraints. Graduates identified a lack of clinician role models for IPC and highlighted the need to address incorrect IPC practices at their training facilities. Faculty also argued that graduates should be trained as 'change agents' with expanded skills in healthcare management, health advocacy and quality improvement. Future studies should determine what additional training is needed to empower medical graduates to become change agents (for healthcare quality improvement) in SA.

Acknowledgements. This research has been supported by the US President's Emergency Plan for AIDS relief (PEPFAR) through Health Resources and Services Administration (HRSA) under the terms of T84HA21652 and via the Stellenbosch University Rural Medical Education Partnership Initiative (SURMEPI). We thank the Stellenbosch University graduates and faculty members for their contribution to this research. Special thanks to Dr Donald Skinner for assistance with development of the master code list and management of the data analysis process using ATLAS.ti.

\section{References}

1. Mehtar S. Understanding Infection Prevention and Control. Cape Town: Juta, 2010:1.

2. National Department of Health. National Core Standards for Health Establishments in South Africa. Pretoria: National Department of Health, 2011.

3. Tavolacci MP, Ladner J, Bailly L, et al. Prevention of nosocomial infection and standard precautions: Knowledge and source of information among healthcare students. Infect Control Hosp Epidemiol 2008;29:642-647. [http://
and and source of information am

dx.doi.org/ $10.1086 / 588683]$
4. Byszewski A, Hendelman W, McGuinty C, Moineau G. Wanted: Role models - medical students' perceptions of professionalism. BMC Med Educ 2012;12:115. [http://dx.doi.org/10.1186/1472-6920-12-115]

5. Frank J. The CanMEDS 2005 physician competency framework. Better standards. Better physicians. Better care Ottawa: The Royal College of Physicians and Surgeons of Canada, 2005.

6. Ritchie J, Lewis J, eds. Qualitative Research Practice: A Guide for Social Science Students and Researchers. London: Sage Publications, 2003.

7. Bradshaw D, Groenewald P, Laubscher R, et al. Initial Burden of Disease Estimates for South Africa, 2000. Cape Town: South African Medical Research Council, 2003.

8. National Department of Health. The National Health Care Facilities Baseline Audit: National Summary Report. Pretoria: National Department of Health, 2012.

9. Kessler CS, McGuinn M, Spec A, Christensen J, Baragi R, Hershow RC. Underreporting of blood and body fluid exposures among health care students and trainees in the acute care setting: A 2007 survey. Am J Infect Control 2011;39(2):129-134. [http://dx.doi.org/10.1016/j.ajic.2010.06.023]

2011;39(2):129-134. [http://dx.doi.org/10.1016/j.ajic.2010.06.023]
Irby DM. Clinical teaching and the clinical teacher. Med Educ 1986;61(9 Pt 2):35-45.

11. Reddy ST, Farnan JM, Yoon JD, et al. Third-year medical students' participation in and perceptions of Reddy ST, Farnan JM, Yoon JD, et al. Third-year medical ste
unprofessional behaviors. Acad Med 2007;82(10 Suppl):S35-S39.

12. Woloschuk WI, Harasym PH, Temple W. Attitude change during medical school: A cohort study. Med Educ 2004;38(5):522-534. 


\section{Research}

\section{Appendix 1. Master code list for ATLAS.ti qualitative data analysis}

Code-Filter: All

HU: faculty inter merged $1-15$ for analysis

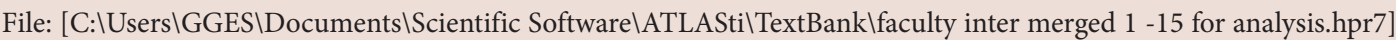

Date/Time: 2013-11-11 11:24:35

CONCERN Challenges to the SA healthcare system: organisation, under resources, lack of facilities

CONCERN Clinical skills v. research skills: tension between these; students not interested in research

CONCERN Dept levels of interest: resistance from dept to teaching these 4 areas; relevance, lack of interest

CONCERN Exposure to risky environments: students faced with risk of TB, crime, etc.

CONCERN Impact of rotations: order of teaching; split between different facilities

CONCERN Information overload: too much info, pressure

CONCERN Lack of skills/knowledge: in dept/competencies/lack of appropriate examples/case studies

CONCERN Lack of time: insufficient faculty members for teaching/load on academics - teaching, patients, research, etc.

CONCERN Overload: too many students/space/facilities/patients for teaching; lack of appropriate context training facilities

CONCERN Poor basic knowledge: difficulty in building on previous learning

CONCERN Problems or concerns (GENERAL): CONCERN Problems with assessment practices: lack of relevant assessment; CONCERN inappropriate assessment models/frameworks from other settings; module outcomes/examination content

CONCERN SA v. external agenda: students training to take up a career overseas

CONCERN Sources of evidence: lack research examples/difficulty accessing info, search practice familiarity

CONCERN Students' lack of interest: anything reflecting students not wanting to engage with subjects

CONCERN Theory v. clinical modules: disconnect and time between teaching certain subjects

IPC GENERAL (anything that doesn't fit in with the other IPC codes)

IPC Knowledge of competencies: discussion of anything related to expected competencies to be achieved by graduates

IPC Measures of evaluation: how the content is examined, both in theory and in practice

IPC Relevance to dept: relevance to their own department

IPC Relevance to faculty: what they thought of the subject teaching in their teaching, clinical and theory

IPC Relevance to medicine/health: relevance of the subject area within greater health context

IPC Teaching in dept: what they thought of the subject teaching in their teaching, clinical and theory

IPC Teaching in faculty: what their perception is of the subject's teaching in the faculty

IPC Understanding of: anything that reflects participants understanding of subject

PARTNERSHIP Barriers to 4 subjects: any reflection on incorporating teaching, resistance to change

PARTNERSHIP Connection to faculty: how they felt connected to the faculty as a whole for their individual disciplines; main campus, other universities PARTNERSHIP Connection to other depts: how they felt connected to other departments, joint teaching/practical work, any shared activities

PARTNERSHIP Co-operation: active co-operation, communication)-

PARTNERSHIP Golden thread: presence of these 4 areas across the curriculum

PARTNERSHIP Integration: integration across the 4 areas with each other (integration into the whole curriculum, clinical teaching)

PARTNERSHIP Partnership in joint process (GENERAL) anything else related

PARTNERSHIP Shared vision: common vision of these 4 areas

PARTNERSHIP Silos where department exists on its own/operates independently, impact of silo thinking on the teaching of 4 areas (compartmentalised subject training)

STUD Future for graduates: anything that happens when the graduate leaves SU

STUD Future in serving poor/rural: anything regarding underserved/rural/poor communities

STUD Preparation of students (GENERAL) STUD resilience training/coping skills: lack of capacity to cope, overload, disorganised teaching

STUD Specialisation v. generalised training: anything touching on that theme

STUD Student ethical practice: patient-centredness, ethical treatment of patients, prioritisation of patients

UNIV Changes in training over time: new curriculum v. old experience of faculty; demands for change (what is required to change the curriculum)

UNIV Doctors v. other professional doctors need different approach to training

UNIV Philosophy for training doctor graduate attributes/the ideal physician/health advocate

UNIV Process of degree training: the model of training undergraduates/postgraduates

UNIV Selection of students: who gets selected to do medical training?

UNIV Shared responsibility: lack of ownership/responsibility for university teaching 4 areas 